\title{
The undersampled wireless acoustic sensor network scenario: some preliminary results and open research issues
}

\section{Citation for published version (APA):}

Sommen, P. C. W., \& Janse, K. (2009). The undersampled wireless acoustic sensor network scenario: some preliminary results and open research issues. In Proceedings of the IEEE Pacific Rim Conference on Communications, Computers and Signal Processing, PACRIM 2009, 24 - 27 August 2009, Victoria, Canada (pp. 877-882). Institute of Electrical and Electronics Engineers. https://doi.org/10.1109/PACRIM.2009.5291252

DOI:

10.1109/PACRIM.2009.5291252

Document status and date:

Published: 01/01/2009

\section{Document Version:}

Publisher's PDF, also known as Version of Record (includes final page, issue and volume numbers)

\section{Please check the document version of this publication:}

- A submitted manuscript is the version of the article upon submission and before peer-review. There can be important differences between the submitted version and the official published version of record. People interested in the research are advised to contact the author for the final version of the publication, or visit the $\mathrm{DOI}$ to the publisher's website.

- The final author version and the galley proof are versions of the publication after peer review.

- The final published version features the final layout of the paper including the volume, issue and page numbers.

Link to publication

\section{General rights}

Copyright and moral rights for the publications made accessible in the public portal are retained by the authors and/or other copyright owners and it is a condition of accessing publications that users recognise and abide by the legal requirements associated with these rights.

- Users may download and print one copy of any publication from the public portal for the purpose of private study or research.

- You may not further distribute the material or use it for any profit-making activity or commercial gain

- You may freely distribute the URL identifying the publication in the public portal.

If the publication is distributed under the terms of Article 25fa of the Dutch Copyright Act, indicated by the "Taverne" license above, please follow below link for the End User Agreement:

www.tue.nl/taverne

Take down policy

If you believe that this document breaches copyright please contact us at:

openaccess@tue.nl

providing details and we will investigate your claim. 


\title{
The undersampled wireless acoustic sensor network scenario: Some preliminary results and open research issues
}

\author{
Piet Sommen \\ Technische Universiteit Eindhoven (TU/e) \\ Department of Electrical Engineering \\ Kees Janse \\ Philips Research Laboratories \\ Digital Signal Processing Group \\ Eindhoven, The Netherlands \\ p.c.w.sommen@tue.nl
}

\begin{abstract}
Advances in hardware technology pave the way to small, low power wireless sensor devices, such as wireless microphones. This makes it possible to use a large number, i.e. thousands, of microphones at positions where it is not feasible to put wired microphones, creating an enormous potential for improved flexibility and performance within a transparent acoustic communication context. In order to reduce battery consumption, each of the wireless microphones has to be sampled much below the Nyquist sample rate. In this paper we will discuss some preliminary results and open research issues that are involved in such an Undersampled Wireless Acoustic Sensor (UWAS) network scenario.
\end{abstract}

\section{Introduction}

In acoustic communication systems people want to create a virtual acoustic communication link that gives conversation partners the impression of being in the same acoustic environment. Besides providing quality and robustness Transparent Acoustic Communication (TAC) systems should exploit the growing computer power to design more flexible systems in which an acoustic interface is built that on the one hand perfectly acquires audio signals, such as speech and sound, and yet allows people to move around freely without wearing or holding a microphone. For this reason we have seen an enormous amount of research in the recent past in sensor arrays (an array of microphones) that can deal with multiple source signals, multiple microphones, multiple loudspeakers running in real time on one or more digital signal processing cores. Although sensor arrays yield a higher performance than single-sensor systems, their performance is limited by the fact that up to now typically a static configuration has been considered, where the position of the array is fixed, the number of sensors is fixed and rather small, and all signal processing is performed on one (eventually multicore) central processor. Within a TAC context, a more flexible scenario in a meeting room is indicated in a.o. [1], where many meeting participants bring portable devices such as laptops, mobiles and PDA's. The microphones of all these different devices form an ad hoc network of distributed microphone arrays. Such an ad hoc array, which will be indicated in this paper as Distributed Portable Acoustic Sensor (DPAS) network, is much more dynamic than the existing fixed acoustic sensor arrays, since the position of the sensors is not exactly known and may even vary in time.

In this paper we will discuss another scenario of distributed microphone arrays within a TAC context. This scenario is based on the observation that advances in hardware technology pave the way to extremely small, low power wireless sensor devices with limited on-board processing capability, enabling individual devices to perform simple processing tasks and to communicate over a short range. Within this context a Micro Electro Mechanical (MEM) based wireless acoustic sensor (microphone) can afford increased flexibility in installation options and can be manufactured in bulk quantity using processes developed by the silicon wafer integrated circuit microchip industry. Due to energy constraints of battery-powered devices, power aware signal processing methods are needed. This can be achieved by sampling each of the wireless acoustic sensors (much) below the Nyquist sample rate, since it is known from literature [2] ${ }^{1}$ that power consumption is linear proportional to the sample frequency of an A/D convertor. Thus the lower the sample frequency of the A/D the lower the battery consumption. This approach opens the door to the possibility of using a large number, i.e. thousands, of low cost undersampled wireless acoustic sensors at positions where it

\footnotetext{
${ }^{1}$ p.50: $P=\alpha f C V_{D D}^{2}$, with $P$ power consumption of any digital block, $V_{D D}$ supply voltage, $C$ total capacity that needs to be switched, $f$ is clock frequency and $\alpha$ parameter expressing the average activity of the gates
} 
is not feasible to put wired microphones, creating an enormous potential for improved flexibility and performance within a TAC context. The dynamic character of such an Undersampled Wireless Acoustic Sensor (UWAS) network is obtained by the fact that it contains a huge amount of sensors from which only a time-varying subset of sensors will produce useful information and will be used. Due to many conceptual differences with a fixed array of sensors, novel array signal processing algorithms need to be developed for the UWAS scenario accounting for different undersampled wireless acoustic sensors, dynamic array configuration, synchronization between the devices, distributed and collaborative processing. This paper will give a general description of the UWAS scenario. Furthermore we discus some preliminary results and some open research topics.

\section{Basic UWAS scenario}

The basic UWAS scenario is depicted in Fig. 1. This

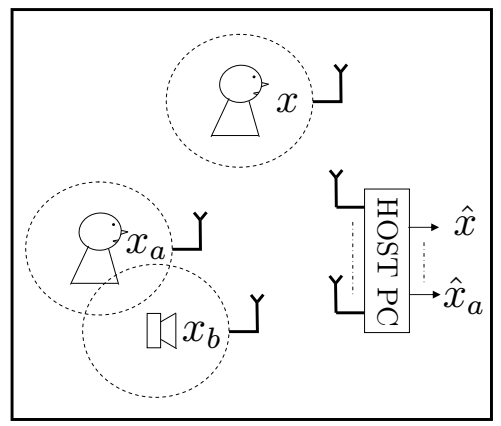
Figure 1. Basic UWAS scenario consisting of
different clusters of acoustic sensors.

figure shows a typical meeting room in which we encounter different audio sources, e.g. speech $\left(x, x_{a}\right)$ and sound $\left(x_{b}\right)$ signals. Some of these sources are desired (e.g. $x$ and $x_{a}$ ) while others may be disturbing (undesired) ones (e.g. $x_{b}$ ). Most of the sources have a non-stationary character and can move around in the room. All the sources are band limited signals with maximum frequency $f_{\max }=1 / 2 \pi T_{0}[\mathrm{~Hz}]$. The meeting room contains a large number, i.e. thousands, of wireless acoustic sensors. These sensors are anywhere in the room, e.g. behind the wall-paper or within the paint on the walls. Only a time-varying subset of sensors will produce useful information. Such subsets are denoted in the figure by dashed circles. Each dashed circle contains a cluster of different undersampled wireless acoustic sensors. Each of these clusters has a wireless transmission link with a host computer. A cluster may sense one signal (e.g. $x$ ) or a mixture of signals (e.g. $x_{a}$ and $x_{b}$ ). Adaptive algorithms that run on the host $\mathrm{PC}$ combine all the incoming sensed signals and produce desired signals, e.g. $\hat{x}$ and $\hat{x}_{a}$ and suppress undesired signals (e.g. $x_{b}$ ). One such cluster of $L$ different undersampled wireless acoustic sensors that senses one source signal $x$ is depicted in Fig. 2. Signal

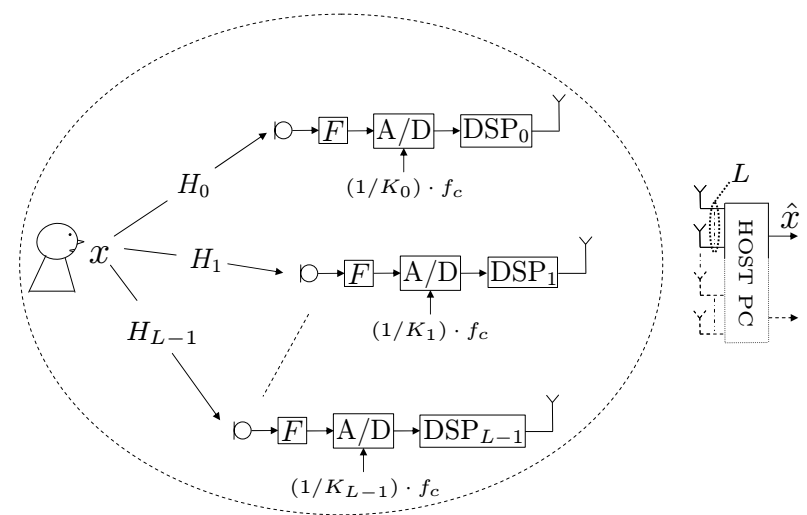

Figure 2. Cluster of $L$ different undersampled wireless acoustic sensors that senses one source signal $x$.

$x$ arrives via $L$ different acoustic transfer functions $H_{i}$, for $i=0,1, \cdots, L-1$, at $L$ different wireless acoustic sensors. Each sensor contains one microphone. The input signal of this microphone is first filtered by an analog prefilter $F$ with cut-off frequency $\left|f_{c}\right|>f_{\max }$. The power consumption of each of the wireless acoustic sensors is limited by using a sample rate $\left(1 / K_{i}\right) \cdot f_{c}$, for $i=0,1, \cdots, L-1$, with $K_{i}$ an integer number. Thus each of the sensors contains samples of an aliased version of the source signal $x$. Each sensing device contains a limited amount of computing power, denoted by the boxes $D S P_{i}$, for $i=0,1, \cdots, L-1$, creating the possibility of distributed processing. Collaborative processing can be obtained by creating a transmission link between different sensing devices. The aliased and eventually pre-processed signal samples are transmitted to a host computer which combines $L$ of these (aliased) UWAS signal samples and calculates a reconstruction $\hat{x}$ of the original source signal $x$. In the following paragraph we will derive a reconstruction structure for a simplified cluster that senses one source signal.

\section{Reconstruction structure for a cluster that senses one source signal}

In this section we will show how a source signal $x$ can be reconstructed in the host $\mathrm{PC}$, when using a simplified setup of a cluster of $L$ acoustic sensors as depicted in Fig. 2. In this simplified setup we make the following assumptions: a) The analog prefilter $F$ is an ideal low pass filter 
with cut-off frequency $\left|f_{c}\right|>f_{\max }$, b) The acoustic transfer functions $H_{l}$ are different, c) All $L$ A/D convertors sample at the same rate $(1 / K) \cdot f_{c}$, with $K$ a natural number $\geq 1$, d) The sensors do not perform any further processing and finally e) The wireless transmission is assumed to be ideal. We will make our further derivation completely in



Figure 3. Discrete-time model of a cluster of $L$ factor $K$ undersampled wireless acoustic sensors that senses one source signal.

the discrete-time domain. For this we need a discrete-time model of the cluster of $L$ factor $K$ undersampled wireless acoustic sensors, which is depicted in Fig. 3. In this figure we used $T_{s}=K \cdot T_{0}$. The frequency response of each of the acoustic transfer functions is given by $H_{l}\left(\mathrm{e}^{\mathrm{j} \theta}\right)$, where $\theta=2 \pi f \cdot T_{0}$ is the normalized discrete-time frequency with period $2 \pi$. It was shown in [3] that the derivation can be simplified by using an alternative model that uses a modulation and demodulation operator. For branch index $l$, with $l=0,1, \cdots, L-1$, this is depicted in Fig. 4. In the upper

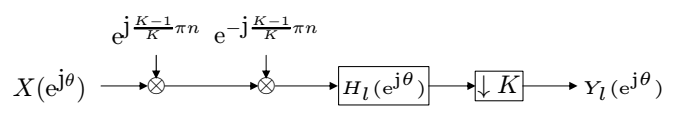

\section{I5}



Figure 4. Alternative model of one branch $l$ of discrete-time model of a cluster that senses one source signal.

part of this figure we have applied a modulation operator to the input signal. The result is that the frequency response of the input signal is shifted over over $\frac{K-1}{K} \pi$ [rad]. This modulation operator is followed by a demodulation operator, which shifts the frequency response of the input signal over the same amount in the opposite direction. In the lower part of this figure, the demodulation operator is first moved over the filter $H_{l}\left(\mathrm{e}^{\mathrm{j} \theta}\right)$, resulting in a shifted version:

$$
H_{s, l}\left(\mathrm{e}^{\mathrm{j} \theta}\right)=H_{l}\left(\mathrm{e}^{\mathrm{j} \theta} \cdot W_{K}^{\frac{K-1}{2}}\right)
$$

with the twiddle factor $W_{K}=\mathrm{e}^{-\mathrm{j} \frac{2 \pi}{K}}$. Finally the demodulation operator is moved over the down-sampling operator which results in the following simple demodulation operator: $\mathrm{e}^{-\mathrm{j}(K-1) \pi n}=(-1)^{(K-1) \cdot n}$. Furthermore the frequency response of the shifted input can be written as:

$$
X_{s}\left(\mathrm{e}^{\mathrm{j} \theta}\right)=X\left(\mathrm{e}^{\mathrm{j} \theta} \cdot W_{K}^{\frac{K-1}{2}}\right)
$$

Using the standard expression for the factor $K$ downsampler we can now derive for each branch $l=0,1, \cdots, L-1$ the following equation in the frequency domain:

$$
Y_{s, l}\left(\mathrm{e}^{\mathrm{j} \theta}\right)=\frac{1}{K} \sum_{q=-\frac{K-1}{2}}^{\frac{K-1}{2}} H_{l}\left(\mathrm{e}^{\mathrm{j} \theta / K} W_{K}^{-q}\right) \cdot X\left(\mathrm{e}^{\mathrm{j} \theta / K} W_{K}^{-q}\right) .
$$

The frequency response of the output is obtained by:

$$
Y_{l}\left(\mathrm{e}^{\mathrm{j} \theta}\right)=Y_{s, l}\left(\mathrm{e}^{\mathrm{j} \theta} \cdot \mathrm{e}^{\mathrm{j}(K-1) \pi}\right) .
$$

Note that the running index $q$ of the summation in equation (3) is defined by: $q=-\frac{K-1}{2}: 1: \frac{K-1}{2}$ and thus $q$ needs not to be an integer. Combining expression (3) for all branches $l=0,1, \cdots, L-1$ results in the following vector-matrix expression:

$$
\underline{\mathbf{Y}}_{s}\left(\mathrm{e}^{\mathrm{j} \theta}\right)=\frac{1}{K} \cdot \mathbf{H}\left(\mathrm{e}^{\mathrm{j} \theta / K}\right) \cdot \underline{\mathbf{X}}\left(\mathrm{e}^{\mathrm{j} \theta / K}\right)
$$

with:

$$
\begin{aligned}
\underline{\mathbf{X}}\left(\mathrm{e}^{\mathrm{j} \theta / K}\right) & =\left(X\left(\mathrm{e}^{\mathrm{j} \theta / K} W_{K}^{\frac{K-1}{2}}\right), \cdots, X\left(\mathrm{e}^{\mathrm{j} \theta / K} W_{K}^{-\frac{K-1}{2}}\right)\right)^{t} \\
\mathbf{H}\left(\mathrm{e}^{\mathrm{j} \theta / K}\right) & =\left(\underline{\mathbf{H}}_{0}\left(\mathrm{e}^{\mathrm{j} \theta / K}\right), \cdots, \underline{\mathbf{H}}_{L-1}\left(\mathrm{e}^{\mathrm{j} \theta / K}\right)\right)^{t} \\
\underline{\mathbf{H}}_{l}\left(\mathrm{e}^{\mathrm{j} \theta / K}\right) & =\left(H_{l}\left(\mathrm{e}^{\mathrm{j} \theta / K} W_{K}^{\frac{K-1}{2}}\right), \cdots, H_{l}\left(\mathrm{e}^{\mathrm{j} \theta / K} W_{K}^{-\frac{K-1}{2}}\right)\right)^{t} \\
\underline{\mathbf{Y}}_{s}\left(\mathrm{e}^{\mathrm{j} \theta}\right) & =\left(Y_{s, 0}\left(\mathrm{e}^{\mathrm{j} \theta}\right), \cdots, Y_{s, L-1}\left(\mathrm{e}^{\mathrm{j} \theta}\right)\right)^{t}
\end{aligned}
$$

In this equation we used underlined boldface characters for vectors and boldface characters for matrices, while $(\cdot)^{t}$ denotes the transpose of a vector. Finally we have to apply a demodulation operator to each of the $L$ branches, which is expressed in the following vector:

$\underline{\mathbf{Y}}\left(\mathrm{e}^{\mathrm{j} \theta}\right)=\left(Y_{s, 0}\left(\mathrm{e}^{\mathrm{j} \theta} \cdot \mathrm{e}^{\mathrm{j}(K-1) \pi}\right), \cdots, Y_{s, L-1}\left(\mathrm{e}^{\mathrm{j} \theta} \cdot \mathrm{e}^{\mathrm{j}(K-1) \pi}\right)\right)^{t}$ 
Furthermore for different acoustic transfer functions $H_{l}$ the $L \times K$ filter matrix $\mathbf{H}\left(\mathrm{e}^{\mathrm{j} \theta / K}\right)$ of equation (6) is nonsingular. The first step of the reconstruction can be achieved by inverting equation (5), which results in:

$$
\frac{1}{K} \cdot \underline{\mathbf{X}}\left(\mathrm{e}^{\mathrm{j} \theta / K}\right)=\mathbf{G}\left(\mathrm{e}^{\mathrm{j} \theta / K}\right) \cdot \underline{\mathbf{Y}}_{s}\left(\mathrm{e}^{\mathrm{j} \theta}\right)
$$

with

$$
\mathbf{G}\left(\mathrm{e}^{\mathrm{j} \theta / K}\right)=\mathbf{H}^{\dagger}\left(\mathrm{e}^{\mathrm{j} \theta / K}\right)
$$

in which ()$^{\dagger}$ denotes the generalized inverse operation. From this point onwards we use an efficient realization of the synthesis part of a DFT modulated filterbank to reconstruct the original source samples $x\left[n T_{0}\right]$ which are represented in frequency domain as $X\left(\mathrm{e}^{\mathrm{j} \theta}\right)$. The reconstruction makes use of the signal samples of $K$ frequency bands of vector $\underline{\mathbf{X}}\left(\mathrm{e}^{\mathrm{j} \theta / K}\right)$ of equation (8). This, non-causal, reconstruction structure is depicted in Fig. 5. Note that we used

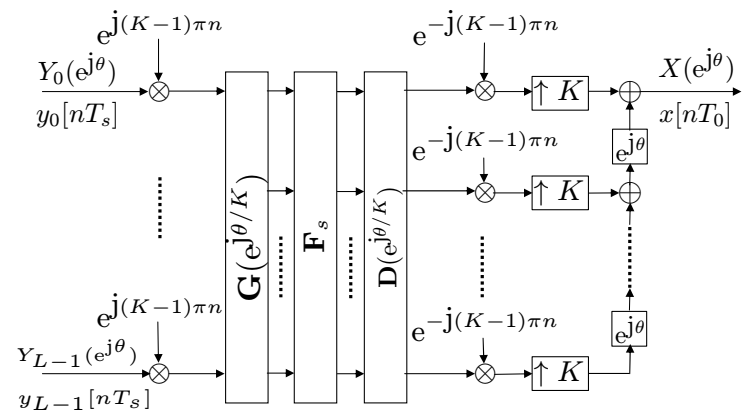

Figure 5. Reconstruction structure (noncausal) for a cluster of $L$ factor $K$ undersampled wireless acoustic sensors that senses one source signal.

a 'shifted' DFT matrix $\mathbf{F}_{s}$ that is defined as:

$$
\begin{aligned}
\mathbf{F}_{s} & =\left(\underline{\mathbf{W}}_{K}^{0}, \cdots, \underline{\mathbf{W}}_{K}^{K-1}\right)^{t} \\
\underline{\mathbf{W}}_{K}^{k} & =\left(W_{K}^{-\frac{K-1}{2} \cdot k}, \cdots, W_{K}^{\frac{K-1}{2} \cdot k}\right)^{t} .
\end{aligned}
$$

Efficiency is achieved by implementing the prototype filter that is used for the synthesis part of the DFT modulated filterbank as polyphase filters in each of the $K$ branches. If furthermore the prototype filter is assumed to be an ideal low pass filter with cut-off frequency $|\pi / K|$ the polyphase decomposed prototype filters reduce to fractional delays. This is represented in the diagonal fractional delay matrix $\left(\mathrm{e}^{\mathrm{j} \theta / K}\right)$ which is, in the ideal case, defined as:

$$
\left(\mathrm{e}^{\mathrm{j} \theta / K}\right)=\operatorname{diag}\left\{\mathrm{e}^{-\mathrm{j} 0 \cdot \theta / K}, \cdots, \mathrm{e}^{-\mathrm{j}(K-1) \cdot \theta / K}\right\} .
$$

Furthermore it noted here that we used in Fig. 5 the symbol $\mathrm{e}^{\mathrm{j} \theta}$ to represent a (non-causal) delay of $T_{0}$ [sec].

\section{Observations:}

1. The reconstruction structure of Fig. 5 reconstructs the uniform Nyquist signal samples of a virtual microphone at the position of sensor 0 by using factor $K$ sub-sampled microphones at $L$ different positions.

2. In a fixed array of sensors we are used to work with the signal samples $x\left[n T_{0}\right]$, with frequency response $X\left(\mathrm{e}^{\mathrm{j} \theta}\right)$. In the UWAS case this signal is, as a result of the factor $K$ downsamplers, split into $K$ subband signals samples, with frequency response $X\left(\mathrm{e}^{\mathrm{j} \theta / K}\right.$. $\left.W_{K}^{\frac{K-1}{2} \cdot q}\right)$. As a result of this the reconstruction has to cope with a mixture of these $K$ subband signals.

In order to obtain more physical insight into the reconstruction structure we will discuss in the following paragraph the result for the case when the acoustic transfer functions $H_{l}\left(\mathrm{e}^{\mathrm{j} \theta / K}\right)$ represent acoustic delays.

\section{Reconstruction structure for a cluster with acoustic delayed sensor signals}

In this section we will use the results of the previous section to derive the reconstruction structure for a cluster that senses one source signal for the special case that the acoustic transfer functions can be represented by pure acoustic delays, thus $H_{l}\left(\mathrm{e}^{\mathrm{j} \theta}\right)=\mathrm{e}^{-\mathrm{j} \tau_{l} \theta}$. We assume all delays are in the interval $0 \leq \tau_{l}<K$ and are different, thus $\tau_{p} \neq \tau_{q}$. Furthermore, without loss of generality, we use $\tau_{0}=0$. Note furthermore that the delays $\tau_{l}$ need not to be integer valued. The sensor samples $y_{l}\left[n T_{s}\right]$ can now be regarded as a recurrent non-uniform sampling process [4]: a combination of $L$ mutual delayed sequences of uniform discretetime signal samples taken at one $K^{t h}$ of the Nyquist sampling rate. An example of a recurrent nonuniform sampling distribution for the case $K=L=3$ is depicted in Fig. 6. This figure shows a time axis on which the small ver-

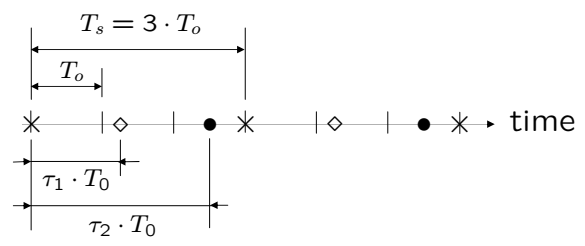

Figure 6. Example of recurrent nonuniform signal samples for $K=L=3$.

tical lines have a distance of $T_{0}$ [sec], the Nyquist period. 
With $K=3$ the under-sampled period equals $T_{s}=3 \cdot T_{0}$ [sec]. Within each under-sampled period of $T_{s}$ [sec] we have $L=3$ samples. The first sample point in each undersampled period is denoted with $\mathrm{a} \times$ at $\tau_{0}=0$ [sec]. The second sample point, denoted with a $\diamond$, has a delay of $\tau_{1} \cdot T_{0}$ [sec] with respect to the first sample point. Finally, the third sample point, denoted with a $\bullet$, has a delay of $\tau_{2} \cdot T_{0}$ [sec] with respect to the first one.

For this special case with acoustic delays we can split $([4,3])$ the filter matrix $\mathbf{H}\left(\mathrm{e}^{\mathrm{j} \theta / K}\right)$ of equation (6) as follows:

$$
\mathbf{H}\left(\mathrm{e}^{\mathrm{j} \theta / K}\right)=\boldsymbol{\Delta}\left(\mathrm{e}^{\mathrm{j} \theta / K}\right) \cdot \mathbf{W}
$$

with $^{2}$ :

$$
\begin{aligned}
\mathbf{W} & =\left(\underline{\mathbf{W}}_{K}^{\tau_{0}}, \cdots, \underline{\mathbf{W}}_{K}^{\tau_{L-1}}\right)^{t} \\
\underline{\mathbf{W}}_{K}^{\tau_{l}} & =\left(W_{K}^{-\frac{K-1}{2} \cdot \tau_{l}}, \cdots, W_{K}^{\frac{K-1}{2} \cdot \tau_{l}}\right)^{t} \\
\Delta\left(\mathrm{e}^{\mathrm{j} \theta / K}\right) & =\operatorname{diag}\left\{\mathrm{e}^{-\mathrm{j} \tau_{0} \theta / K}, \cdots, \mathrm{e}^{-\mathbf{j} \tau_{L-1} \theta / K}\right\}(13)
\end{aligned}
$$

Using this matrix splitting in equation (5) the acoustic sensor model becomes:

$$
\underline{\mathbf{Y}}_{s}\left(\mathrm{e}^{\mathrm{j} \theta}\right)=\frac{1}{K} \cdot \boldsymbol{\Delta}\left(\mathrm{e}^{\mathrm{j} \theta / K}\right) \cdot \mathbf{W} \cdot \underline{\mathbf{X}}\left(\mathrm{e}^{\mathrm{j} \theta / K}\right)
$$

Inverting this equation results in a vector with $K$ subbands of the source signal:

$$
\frac{1}{K} \cdot \underline{\mathbf{X}}\left(\mathrm{e}^{\mathrm{j} \theta / K}\right)=\mathbf{W}^{\dagger} \cdot \boldsymbol{\Delta}^{-1}\left(\mathrm{e}^{\mathrm{j} \theta / K}\right) \cdot \underline{\mathbf{Y}}_{s}\left(\mathrm{e}^{\mathrm{j} \theta}\right)
$$

and the reconstruction structure simplifies to the one that is depicted in Fig. 7. The first step of this scheme is the mod-

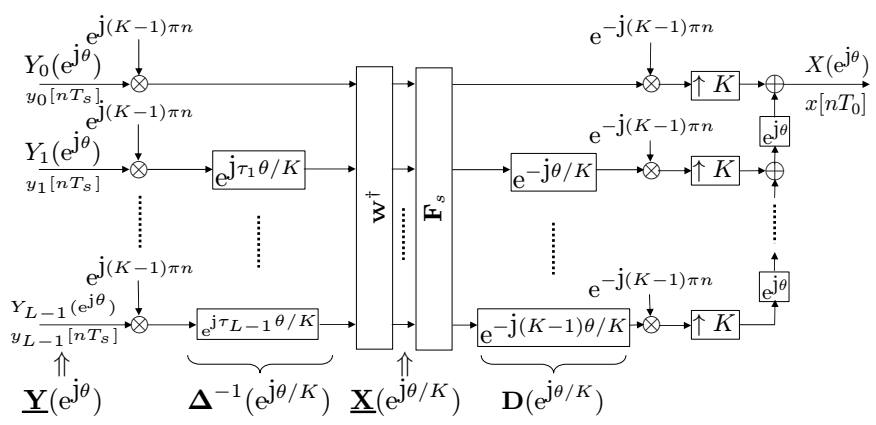

Figure 7. Reconstruction structure (noncausal) for a cluster of $L$ factor $K$ undersampled wireless acoustic sensors that senses one source signal in case of acoustic delays.

\footnotetext{
${ }^{2}$ Matrix $\mathbf{W}$ is the nonuniform equivalent of the DFT matrix $\mathbf{F}_{s}$ as defined in equation(10)
}

ulation of the incoming signals. In order to further process the $L$ parallel signals, a proper time alignment is needed which is taken care of by the inverse of the diagonal filter matrix $\boldsymbol{\Delta}\left(\mathrm{e}^{\mathrm{j} \theta / K}\right)$. Each of the resulting signals contains a mixture of $K$ subbands of the input signal vector $\underline{\mathbf{X}}\left(\mathrm{e}^{\mathrm{j} \theta / K}\right)$. This mixture is de-mixed by the generalized inverse of matrix W. From this point onwards the structure is equivalent to the synthesis part of an efficient DFT modulated uniform filterbank.

\section{Observations:}

1. From the above derived structure it follows that for known and different acoustic delays $\tau_{l}$ the reconstruction is possible for $L=K$, since for such a case the matrix $\mathbf{W}$ is square and non singular. Thus in order to reconstruct the source signal samples at uniform Nyquist rate we need the same amount of non uniform sampling points of the source signal in one pe$\operatorname{riod} T_{s}=K \cdot T_{0}$ compared to the number of uniform sampling points. This is in line with the results of [5].

2. For the very special case when the acoustic delays are known and successive integer values, thus $K=L$ and $\tau_{l}=l$, for $l=0,1, \cdots, L-1$, the values of the signal samples $y_{l}\left[n T_{s}\right]$ represent $L$ successive uniform samples of the original source samples $x\left[n T_{0}\right]$. For this uniform sampled case the reconstruction structure of Fig. 7 can be simplified since we have $\mathbf{F}_{s} \cdot \mathbf{W}^{-1}=\mathbf{I}_{K}$, with $\mathbf{I}_{K}$ the $K \times K$ identity matrix. Furthermore when using an ideal prototype filter for the synthesis part, we also have $\left(\mathrm{e}^{\mathrm{j} \theta / K}\right) \cdot \boldsymbol{\Delta}^{-1}\left(\mathrm{e}^{\mathrm{j} \theta / K}\right)=\mathbf{I}_{K}$. Thus for this uniform sampled case the whole reconstruction structure of Fig. 7 reduces, as expected, to a timeinterleaved structure which consists of a set of $K=L$ parallel up-samplers and (non-causal) delays.

\section{Open research issues}

Due to many conceptual differences with a fixed array of sensors, novel array signal processing algorithms need to be developed for the UWAS scenario. Some of these open research issues are discussed in this section.

One of the first steps to study within the UWAS scenario is a cluster that senses one source, as depicted in Fig. 2. For such a cluster we assume that we have selected a set of $L$ acoustic sensors that sense one source signal $x$. The main question is how to reconstruct the uniform signal samples $\hat{x}\left[n \cdot T_{0}\right]$ at Nyquist rate based on the samples of $L$ undersampled sensor signals. Obviously this study has to be done (similar to section 3 ) with acoustic transfer functions $H_{l}$. However, in order to obtain more insight, it is useful to simplify the acoustic transfer functions in first instance by acoustic delays (similar to section 4). In this simplified case 
the $L$ sensor signals represent $L$ nonuniform samples of the source signal. Some relevant research questions of this non uniform sampling approach are given below:

1. Blind and adaptive reconstruction algorithms: If all acoustic delays are known, we have shown in section 4 that we need $L=K$ acoustic sensors in order to reconstruct the original uniform (Nyquist) signal samples. In a more practical context however the acoustic delays will be unknown and may even vary in time. Thus blind and adaptive reconstruction algorithms are needed. Although such algorithms are not available yet, it is argued here that we can construct a set of equations which can be solved.

The vector-matrix equation (14) of the acoustic sensor model, which consists of $L$ equations, holds for any frequency $\theta_{i}$ in the range $\left|\theta_{i}\right|<\pi$. We can use this equation to construct $L$ equations at $N$ different frequencies, say $\theta_{i}$, with $i=0,1, \cdots, N-1$. For each new frequency $\theta_{i}$ we have $K$ new unknown variables of the signal vector $\underline{\mathbf{X}}\left(\mathrm{e}^{\mathrm{j} \theta_{i}}\right)$. However the $L$ unknown delays are the same for each new frequency $\theta_{i}$. Thus we can create in this way $N \cdot L$ equations in order to calculate $L+N \cdot K$ unknowns. This system of equations can be solved if: $N \cdot L \geq L+N \cdot K$ or equivalently $N \geq L /(L-K)$. From this it follows that we can construct a blind reconstruction scheme by evaluating the $L$ sensor signals at $N$ different frequencies and with $L>K$ acoustic sensors. Note that $L>K$ implies a form of oversampling of the original source signal. A final remark here is that we can reduce the number of equations $N$ by using symmetry properties that exist between the different frequency bands $X\left(\mathrm{e}^{\mathrm{j} \theta / K} W_{K}^{-q}\right)$.

2. Timing ambiguity:

In the development of the reconstruction structure of section 4 , we used the assumption that all delays are in the interval $0 \leq \tau_{l}<K$. This restriction causes a timing ambiguity for delays that are outside this interval.

3. Tuning power consumption:

The battery level of all acoustic sensors can be different. Thus all the subsample factors $\left(1 / K_{l}\right) \cdot f_{c}$ will be different. This implies that the reconstruction structure of section 4 needs to be generalized for such a case.

4. Robustness:

Different issues that are discussed in this paper deal with ideal situations which do not hold in practice. One of these issues is the fact that the reconstruction structures that are depicted in Fig. 5 and Fig. 7 make use of the synthesis part of ideal uniform DFT modulated filterbanks. In practice the used filters will not be ideal and will cause leakage. More robust structures need to be developed.
After the generalization of the above research topics to the more general case of acoustic transfer functions, the next step is to study a cluster that senses different sources. When different source signals are sensed by a cluster of $L$ undersampled acoustic sensors we need to develop new beam forming, source separation and source extraction algorithms. An important part to study of the UWAS scenario is obviously the overall UWAS scenario. Some important topics are: a) Dynamic array configuration: Which timevarying subset of sensors produces useful information? b) Synchronization: The oscillation drift of the A/D conversions of the different acoustic sensors may be different and will cause synchronization errors. c) Distributed and collaborative processing: How can we use the sensor DSP's in order to collaborate or to distribute the total amount of processing?

\section{Conclusions}

In this paper we presented some preliminary results of an UWAS scenario and we posed some open research questions. We do believe that many new array processing algorithms have to be developed in the UWAS scenario. Such algorithms have to account for combining undersampled signal samples, dynamic array configuration, synchronisation between the devices, and distributed and collaborative processing aimed at meeting power and complexity constraints.

\section{References}

[1] Jacek Dmochowski, Zicheng Liu and Phil Clou; "Blind source separation in a distributed microphone meeting environment for improved teleconferencing"; ICASSP conference; Las Vegas, Nevada; 2008; pp 8992

[2] Kathleen Philips; " $\Sigma \Delta$ A/D Conversion for Signal Conditioning"; PhD thesis TU/e; June 2005; ISBN 90 74445-68-3

[3] Piet Sommen and Kees Janse; "On the reconstruction of undersampled wireless acoustic sensor signals"; IWAENC conference; Seattle, USA; september 2008

[4] Piet Sommen, Kees Janse; "On the relationship between uniform and recurrent nonuniform discretetime sampling schemes"; IEEE Trans. on SP; Vol.56, No.10, Part2; Oct. 2008; pp5147 - 5156

[5] Yonina C. Eldar, Alan V. Oppenheim; "Filter reconstruction of bandlimited signals from nonuniform generalized samples"; IEEE Trans. on SP; vol.48, no.10; october 2000; pp 2864 -2875 